Sharif University of Technology
Scientia Iranica
SCIENTIA $\quad \begin{gathered}\text { Transactions D: Computer Science \& Engineering and Electrical Engineering } \\ \text { w }\end{gathered}$

\title{
A new solution approach to supply function equilibrium-based bidding strategy in electricity markets
}

\author{
A. Azadi Hematabadi and A. Akbari Foroud* \\ Faculty of Electrical \& Computer Engineering, Semnan University, Semnan, Iran.
}

Received 26 December 2015; accepted 29 August 2016

\section{KEYWORDS \\ LSF parameterization; Analytical solution; Pool-based electricity markets; \\ Bi-level optimization; Integrated energy and reserve markets.}

\begin{abstract}
This paper presents a new analytical solution method for Supply Function Equilibrium-based (SFE) bidding strategy in electricity markets. It is assumed that every Generation Company (GenCo) has some generation units and bids a Linear Supply Function (LSF) for each of its units to the Independent System Operator (ISO). The problem is modeled as a bi-level optimization problem; in the inner level, ISO clears the market to maximize social welfare; in the outer level, each GenCo tries to maximize its individual welfare. The proposed method is used to solve the outer-level problem using an iterative algorithm, in which LSF coefficients are parameterized. The formulation is developed for both elastic and non-elastic demands for single- and multi-generator cases and also for integrated energy and Spinning Reserve (SR) markets with three models of competitions: $a-, b-$, and $k$-parameterization. Three sample networks are used to evaluate the proposed method. The results show that the proposed method is effective and accurate for GenCos' strategic bidding in electricity markets compared with other optimization algorithms.

(C) 2017 Sharif University of Technology. All rights reserved.
\end{abstract}

\section{Introduction}

Electricity markets have provided opportunities for competing and gaining more revenues for market participants by optimal bidding strategies [1]. This kind of market with imperfect competition is called oligopoly market, and the participants trying to raise market price by their strategic bidding are known as price makers. Generally, there are three main methods for choosing strategic bidding in these oligopoly markets: market price forecasting, rivals' bidding modeling, and game-based rivals' strategic behavior simulating [2].

*. Corresponding author. Fax: +9823 33654089 E-mail addresses: aazadi@semnan.ac.ir (A. Azadi Hematabadi); aakbari@semnan.ac.ir (A. Akbari Foroud)

doi: $10.24200 /$ sci. 2017.4360
Game theory is used as a tool to analyze electricity market, considering the fact that the price makers react to their rivals' strategies to maximize their profit [3]. Based on game theory, several competitive models have been proposed in the literature to analyze the behavior of GenCos' bidding strategies such as Bertrand, Cournot, Stackelberg, and Supply Function Equilibrium (SFE). Only SFE can make a relationship between the price and quantity of GenCo's bid, and only this model is the closest to the actual behavior of players [4]. The SFE was introduced by Klemperer and Mayer [5], but the Linear Supply Function (LSF) was used in [6] and has ever since been used in various papers to model participants' behaviors in the electricity markets.

In [7], the LSF parameterization is summarized into four classifications. Three of these have one degree of freedom for strategic variables, i.e. slope 
parameterization, intercept parameterization, and similar parameterization of slope and intercept. In the last one, with two degrees of freedom, the slope and intercept are parameterized arbitrarily. Although it was explained in [8] that the arbitrary parameterization of the slope and intercept gives more realistic results, using this model is difficult because it may lead to several equilibrium points [9]. In many papers, the first three methods have been used to model the behavior of market players.

GenCos can gain more profit in the market with various products (e.g., energy [8], reserve [10], reactive power [11], and heat [12]). Ancillary services, such as spinning reserves, play a significant role in ensuring the stability of restructured power systems [13]. In the early years of electricity market, energy and ancillary services used to be settled in separate markets; however, there is wide consensus today that energy and reserve should be offered and cleared simultaneously in integrated markets to minimize the overall cost of supplying energy and provision of reserve [14]. In recent years, various papers have dealt with the simultaneous settlement of energy and Spinning Reserve (SR) [15-19]. In [18] a thorough literature review on joint energy and reserve markets modeling is presented.

The main purpose of the power system restructuring is to maximize the social welfare by increasing competition in the market, and this allows the market participants to maximize their own individual profits [20]. For an individual player, searching for the optimized bidding strategy is based on the information about or estimation of the rivals' actions. Recently, bilevel optimization method has been used to optimize the producers' bidding strategy. In the outer level, each GenCo tries to maximize its individual welfare by adjusting its supply function parameters; in the inner level, ISO tries to maximize the social welfare [21]. To solve these two problems, authors have utilized various tools of optimization such as BA algorithm [3], Lagrangian relaxation [17], Q-learning [22], and genetic algorithm [23].

The contribution of this paper is presenting a new analytical solution method for producers' bidding strategy problem in oligopoly power markets. In this method, a new iterative algorithm has been used to solve the outer level of the bi-level optimization problem. Considering the rivals' logical action, LSF parameters can be adjusted using the proposed method. The proposed method is developed for single-generator and multi-generator GenCos strategic biddings in integrated energy and reserve markets with both elastic and non-elastic demands and with resource constraint. Some of the advantages of the proposed method are mentioned in the following, which are proven and shown by an example in the numerical result section:
- Its simplicity compared with other solution tools such as GA;

- Not suffering from the problems of convergence;

- High convergence speed with low iteration number.

The rest of the paper are structured as follows: formulation of market settlement is discussed in Section 2 ; the proposed method is explained in Section 3; the numerical results are shown in Section 4; the paper is concluded in Section 5 .

\section{Market settlements}

\subsection{Market assumptions}

Imagine that there are $G$ numbers of GenCos in the market; each GenCo has $g$ generation units (generators), and these units can simultaneously bid in both energy and spinning reserve markets. First, the demand is assumed to be non-elastic (in the next section, the relationships are extended to elastic demand cases). The overall costs of energy and reserve services for unit $k$ of GenCo $i$ are respectively shown by convex quadratic functions as follows:

$$
\begin{array}{rlrl}
C_{i k}^{e} & =\frac{1}{2} a_{i k} q_{i k}^{2}+b_{i k} q_{i k}, & & a_{i k}>0, \\
C_{i k}^{r}=\frac{1}{2} \rho_{i k} R_{i k}^{2}+\vartheta_{i k} R_{i k}, & & \rho_{i k}>0 .
\end{array}
$$

According to Eqs. (1) and (2), the Marginal Cost (MC) of energy and reserve for unit $k$ of GenCo $i$ can be defined:

$$
\begin{aligned}
& \mathrm{MC}_{i k}^{e}=a_{i k} q_{i k}+b_{i k}, \\
& \mathrm{MC}_{i k}^{r}=\rho_{i k} R_{i k}+\vartheta_{i k},
\end{aligned}
$$

where $q_{i k}$ and $R_{i k}$ are the quantities for the energy and reserve markets, respectively. Due to the low number of players in electricity markets, these markets' performances are based on oligopoly markets. In oligopoly markets, as mentioned in introduction, some players can affect the Market-Clearing Price (MCP). In such a case, MCP and quantity dispatched to each GenCo depend not only on the decisions made by a firm, but also on its rivals' actions in the markets.

Imagine that GenCo $G_{i}$ bids a Linear Supply Function (LSF) for each of its own units to the Independent System Operator (ISO) in the form of Eqs. (5) and (6) to participate in the energy and reserve markets, respectively:

$$
\widehat{\mathrm{MC}}_{i}^{e}=\left\{\begin{array}{l}
\alpha_{i 1} q_{i 1}+\beta_{i 1}, \\
\alpha_{i 2} q_{i 2}+\beta_{i 2}, \\
\vdots \\
\alpha_{i g_{i}} q_{i g_{i}}+\beta_{i g_{i}}
\end{array}\right.
$$




$$
\widehat{\mathrm{MC}}_{i}^{r}=\left\{\begin{array}{l}
\gamma_{i 1} R_{i 1}+\theta_{i 1} \\
\gamma_{i 2} R_{i 2}+\theta_{i 2} \\
\vdots \\
\gamma_{i g_{i}} R_{i g_{i}}+\theta_{i g_{i}}
\end{array}\right.
$$

where $\alpha_{i k}$ and $\beta_{i k}$ are the strategic variables of unit $k$ in the energy market, and $\gamma_{i k}$ and $\theta_{i k}$ are its strategic variables for reserve market. ISO clears energy and reserve markets based on GenCos' bidding, demand, and reserve requirements for system security.

\subsection{The ISO problem}

The goal of ISO is to maximize the social welfare. If demand is non-elastic, the maximization of the social welfare equals the minimization of the cost of energy supply and reserve services. In [24], a strategic game model in the form of a Mathematical Program with Equilibrium Constraints (MPEC) is introduced, in which supplier's bidding problem is formulated as a bi-level optimization problem. In the inner level, ISO clears the market based on the suppliers' bids to maximize the social welfare. Based on that method, Bautista et al. [15] has presented an oligopoly model for the integrated markets of energy and reserve, where the bids are in the form of constant prices.

In this paper, considering the settlement mechanism of the uniform pricing, the ISO problem is solved through the MPEC model, and the results are used to solve the GenCo's problem (i.e., outer level). For simplicity, in this section, it is assumed that every GenCo has only one generator; but, in the next section, the formulation will be extended to the multi-generator case. Accordingly, if the bids of GenCos for energy and reserve markets are in the form of Eqs. (5) and (6), the formulation of simultaneous settlement of these two markets for the non-elastic demand cases will be:

$$
\begin{array}{ll}
\min & \sum\left(0.5 \alpha_{j} q_{j}^{2}+\beta_{j} q_{j}+0.5 \gamma_{j} R_{j}^{2}+\theta_{j} R_{j}\right), \\
\text { s.t. } & q_{i}+R_{i} \leq q_{i}^{\max }, \quad q_{i} \geq 0 \\
& \sum_{j=1}^{n} q_{j}=Q_{D}, \\
& \sum_{j=1}^{n} R_{j} \geq Q_{\mathrm{SR}} .
\end{array}
$$

Eq. (7) shows the overall cost of producing energy and spinning reserve services that ISO calculates based on the GenCos' bidding. Eq. (8) shows the GenCos' capacity limit of energy and reserve services, and Eq. (9) shows the supply and demand equality constraint in the energy market. Eq. (10) shows the inequality constraint in the reserve market where $Q_{\mathrm{SR}}$ is the minimum spinning reserve capacity requirements of the network determined through some criteria by ISO. In the literature, different methods have been proposed to determine $Q_{\mathrm{SR}}$, the most famous of which are: a fraction of the total demand $\left(Q_{\mathrm{SR}}=k_{s} \cdot Q_{D}\right)$, such as the Spain system, and the capacity of the biggest available generation unit or transmission line $\left(Q_{\mathrm{SR}}=\max \left\{q_{1}^{\max }, \cdots, q_{n}^{\max }, F_{1}^{\max }, \cdots, F_{n}^{\max }\right\}\right)$, such as the Ontario system. In addition, different values have been proposed for $k_{s}$, like $14 \%$ in [25] and $7 \%$ in [16]. In this paper, SR requirement for system security is considered $10 \%$ of the total demand (i.e., $\left.Q_{\mathrm{SR}}=\frac{1}{10} \times Q_{D}\right)$ like [26].

By writing the Karush-Kuhn-Tucker condition for primary problems (7)-(10) and using dual variables $\lambda_{e}$, $\lambda_{r}$, and $\mu_{i}$, we have:

$$
\begin{aligned}
& 0 \leq q_{i}^{\max }-q_{i}-R_{i} \perp \mu_{i} \geq 0 \\
& 0 \leq q_{i} \perp \mu_{i}-\lambda_{e}+\beta_{i}+\alpha_{i} \cdot q_{i} \geq 0 \\
& 0 \leq R_{i} \perp \mu_{i}+-\lambda_{r}+\theta_{i}+\gamma_{i} \cdot R_{i} \geq 0 \\
& 0 \leq \lambda_{r} \perp \sum_{j=1}^{n} R_{j}-Q_{\mathrm{SR}} \geq 0 \\
& \lambda_{e} \text { free } \sum_{j=1}^{n} q_{j}-Q_{D}=0
\end{aligned}
$$

If index $i(i \in I)$ is used for the constrained GenCos, according to Relations (12) and (13), we have:

$$
\begin{aligned}
& \mu_{i}-\lambda_{e}+\beta_{i}+\alpha_{i} q_{i}=0, \\
& \mu_{i}-\lambda_{r}+\theta_{i}+\gamma_{i} R_{i}=0 .
\end{aligned}
$$

For other non-constrained GenCos $(k \in\{G-I\})$ whose coefficient $\mu_{k}$ is equal to zero, we have:

$$
\begin{aligned}
& -\lambda_{e}+\beta_{k}+\alpha_{k} q_{k}=0, \\
& -\lambda_{r}+\theta_{k}+\gamma_{k} R_{k}=0 .
\end{aligned}
$$

By solving Eqs. (16) and (18) for the energy market, we have:

$$
\begin{aligned}
q_{i} & =\frac{\lambda_{e}-\beta_{i}-\mu_{i}}{\alpha_{i}}, \\
q_{k} & =\frac{\lambda_{e}-\beta_{k}}{\alpha_{k}} \quad k \neq i .
\end{aligned}
$$

Substituting Eqs. (20) and (21) into Eq. (9) yields:

$$
\sum_{j=1}^{I}\left(\frac{\lambda_{e}-\beta_{j}-\mu_{j}}{\alpha_{j}}\right)+\sum_{j=I+1}^{G}\left(\frac{\lambda_{e}-\beta_{j}}{\alpha_{j}}\right)=Q_{D} .
$$

According to Eqs. (20)-(22), the energy market equi- 
librium is as follows:

$$
\begin{gathered}
\lambda_{e}=\frac{Q_{D}+\sum_{j=1}^{G}\left(\frac{\beta_{j}}{\alpha_{j}}\right)+\sum_{j=1}^{I}\left(\frac{\mu_{j}}{\alpha_{j}}\right)}{\sum_{j=1}^{n}\left(\frac{1}{\alpha_{j}}\right)}, \\
q_{i}=\frac{Q_{D}+\sum_{j=1}^{G}\left(\frac{\beta_{j}-\beta_{i}-\mu_{i}}{\alpha_{j}}\right)+\sum_{j=1}^{I}\left(\frac{\mu_{j}}{\alpha_{j}}\right)}{\sum_{j=1}^{n}\left(\frac{\alpha_{i}}{\alpha_{j}}\right)} \\
q_{k}=\frac{Q_{D}+\sum_{j=1}^{G}\left(\frac{\beta_{j}-\beta_{k}}{\alpha_{j}}\right)+\sum_{j=1}^{I}\left(\frac{\mu_{j}}{\alpha_{j}}\right)}{\sum_{j=1}^{n}\left(\frac{\alpha_{k}}{\alpha_{j}}\right)} \quad(k \neq i) .
\end{gathered}
$$

Similarly, SR market equilibrium can be obtained as:

$$
\begin{aligned}
& \lambda_{r}= \frac{Q_{\mathrm{SR}}+\sum_{j=1}^{G}\left(\frac{\theta_{j}}{\gamma_{j}}\right)+\sum_{j=1}^{I}\left(\frac{\mu_{j}}{\gamma_{j}}\right)}{\sum_{j=1}^{n}\left(\frac{1}{\gamma_{j}}\right)}, \\
& R_{i}=\frac{Q_{\mathrm{SR}}+\sum_{j=1}^{G}\left(\frac{\theta_{j}-\theta_{i}-\mu_{i}}{\gamma_{j}}\right)+\sum_{j=1}^{I}\left(\frac{\mu_{j}}{\gamma_{j}}\right)}{\sum_{j=1}^{n}\left(\frac{\gamma_{i}}{\gamma_{j}}\right)}, \\
& R_{k}=\frac{Q_{\mathrm{SR}}+\sum_{j=1}^{G}\left(\frac{\theta_{j}-\theta_{k}}{\gamma_{j}}\right)+\sum_{j=1}^{I}\left(\frac{\mu_{j}}{\gamma_{j}}\right)}{\sum_{j=1}^{n}\left(\frac{\gamma_{i}}{\gamma_{j}}\right)} \quad(k \neq i),
\end{aligned}
$$

where Eqs. (23) and (26) show the energy and reserve market prices, respectively. In addition, Eqs. (24) and (27) show the quantities of power and SR allocated to constrained generator $i$, respectively; (25) and (28) show the quantities of power and reserve allocated to non-constrained generator $k$, respectively. In these equations, coefficient $\mu_{i}$ is the marginal cost of the capacity constraint. For non-constrained GenCos, $\mu_{i}$ equals zero, while, for the constrained GenCos, it is a positive value that increases the price of energy and reserve services according to Eqs. (23) and (26). In fact, what has caused GenCo $i$ to be constrained is its lower bidding price compared to its rivals; in other words, to increase the social welfare, ISO gives the generation priority to cheaper producers. When cheaper GenCo $i$ has to have generation at its maximum capacity, the share of more expensive Gen Cos increases because demand is not elastic; therefore, the market price rises. The way to calculate coefficient $\mu_{i}$ is shown in $[17]$.

\subsection{The GenCo problem}

The revenue of a GenCo equals the difference between the income from sales and the generation cost. The income obtained from selling energy and reserve services depends on the market settlement mechanism. To gain more revenue, the strategic players maximize their expected revenue through supply function parameterization, thereby manipulating the prices in the two markets of energy and spinning reserve.

Considering the settlement mechanism of uniform payment for both markets of energy and reserve, the income of company $k$ is:

$$
\operatorname{Rev}_{k}=P^{e} \cdot q_{k}+P^{r} \cdot R_{k} .
$$

If the total cost of both energy and reserve for GenCo $k$ is equal to the sum of Eqs. (1) and (2); according to Eq. (29), the profit is:

$$
\Omega_{k}=P^{e} \cdot q_{k}+P^{r} \cdot R_{k}-\frac{1}{2} a_{k} q_{k}^{2}-b_{k} q_{k} \frac{1}{2} \rho_{k} R_{k}^{2}-\vartheta_{k} R_{k} .
$$

Therefore, according to the results of the ISO problem, each GenCo tries to maximize its expected profit through an optimization process by considering the constraints:

$$
\begin{aligned}
& \max \left\{\Omega_{k}\right\}, \\
& \text { s.t. } \quad \text { Constraints }(8-10) .
\end{aligned}
$$

In this paper, assuming that all players know the results of market settlement, each player optimizes its bidding by simulating a non-cooperative game with complete information without imperfect competition. In other words, although the player is not aware of the other player's bidding, he/she can adjust its bidding by having some information such as the possible actions and strategies and also the payoff function of its rivals. Accordingly, we can consider the flowchart in Figure 1 to determine the optimal bidding strategy of every strategic player, which was similarly used in [24]. This game continues until none of the strategic players considers the change in their bidding strategy useful. This is where we say the Nash equilibrium has been established [1].

\section{The proposed method}

When market competition model is based on LSF, there are four models of parameterization, which are classified in the following [7]:

1. a-parameterization: Where each strategic player tries to maximize its expected profit by parameterizing the slope of supply function. In such a case, the intercept is fixed and is usually kept at the real amount of $\mathrm{MC}$ (i.e., $b_{i}$ ). In this case, the strategic variable of every strategic player is $\alpha$; 


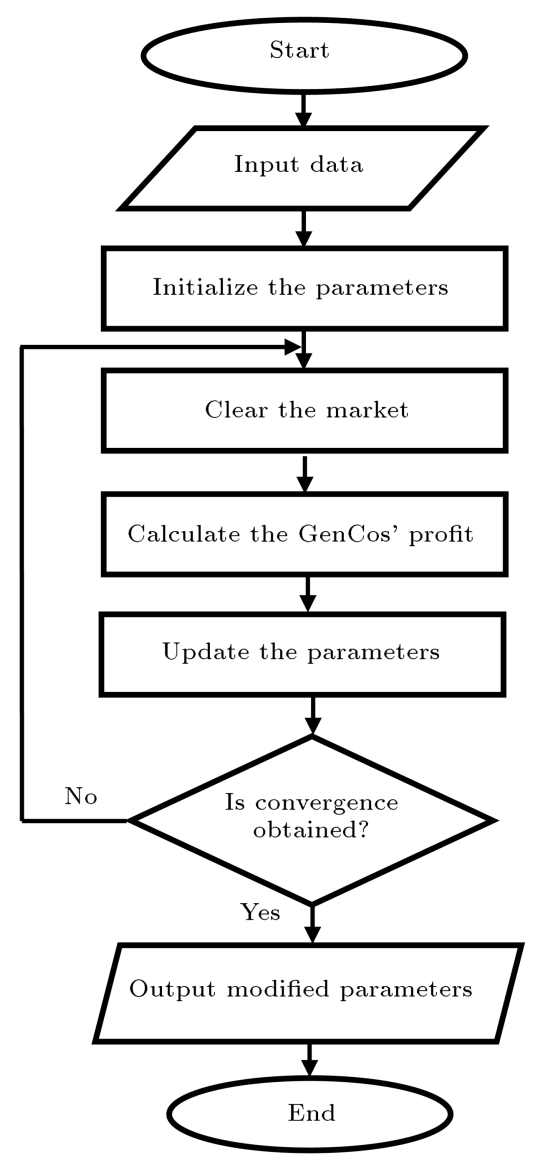

Figure 1. Flowchart of the optimal bidding strategy.

2. b-parameterization: In this case, the strategic player adjusts the intercept of LSF in order to get more payoff. The slope of LSF is fixed and is usually kept at the amount of MC. In this case, the strategic variable of each strategic player is $\beta$;

3. k-parameterization: In this case, each strategic player changes the slope and intercept similarly, i.e. $\alpha_{i}=k_{i} . a_{i}$ and $\beta_{i}=k_{i} . b_{i}$. GenCo's decision variable is coefficient $k_{i}$;

4. (a,b)-parameterization: In this case, the slope and intercept of LSF is manipulated arbitrarily. This model has not been studied in this paper and will be dealt with by future studies.

First, in Sections 3.1 and 3.2, formulations of the proposed method for parameterizing of the LSF coefficients to bid in the energy market are explained for the two cases of non-constrained and constrained GenCos, respectively. Then, in Section 3.3, the obtained formulations are generalized for the joint market of energy and reserve. In addition, the relationships for the elastic demand cases are developed in Section 3.4.

\subsection{Energy market without constraint}

In this paper, a new iterative solution method for LSF parameterization is presented, in which LSF coeffi- cients are adjusted optimally. To do so, we take the derivative of profit function according to these coefficients. By setting the first-order derivative at zero, the relationships are obtained, which are presented in the following subsections.

\subsubsection{Slope parameterization}

In this case, it is assumed that every GenCo only parameterizes the slope of its MC. The supply function and the expected payoff of GenCo $k$ in the energy market are respectively as follows:

$$
\begin{aligned}
& \widehat{\mathrm{MC}}_{k}^{e}=\alpha_{k} q_{k}+b_{k}, \\
& \Omega_{k}=P^{e} \cdot q_{k}-\frac{1}{2} a_{k} q_{k}^{2}-b_{k} q_{k} .
\end{aligned}
$$

Taking derivative of Eq. (33) according to slope of LSF yields:

$$
\frac{\partial \Omega_{k}}{\partial \alpha_{k}}=q_{k} \frac{\partial P^{e}}{\partial \alpha_{k}}+\left(P^{e}-a_{k} q_{k}-b_{k}\right) \frac{\partial q_{k}}{\partial \alpha_{k}} .
$$

According to Eqs. (18) and (20) and by taking derivative of $P^{e}$ and $q_{k}$ based on $\alpha_{k}$, we will have Eqs. (35) and (36), respectively, as follows:

$$
\begin{aligned}
& \frac{\partial P^{e}}{\partial \alpha_{k}}=\left(\frac{1}{\sum_{j=1}^{G} \frac{\alpha_{i}}{\alpha_{j}}}\right) \cdot q_{k}, \\
& \frac{\partial q_{k}}{\partial \alpha_{k}}=-\left(\frac{\sum_{\substack{j=1 \\
j \neq i}}^{G} \frac{1}{\alpha_{j}}}{\sum_{j=1}^{G} \frac{\alpha_{i}}{\alpha_{j}}}\right) \cdot q_{k}
\end{aligned}
$$

By substituting Eqs. (35) and (36) into Eq. (34) and setting it as zero, we can reach a recursive relationship such as Eq. (37) to parameterize the slope in an iterative game according to the flowchart of Figure 1:

$$
\alpha_{k}^{(t+1)}=a_{k}+\frac{1}{\sum_{\substack{j=1 \\ j \neq k}}^{G} \frac{1}{\alpha_{k}^{(t)}}}
$$

where $t$ is the number of iterations. For each iterative method, there is the need for both initial point and stop condition. To initiate the process, we can use the real value of slope of MC. Also, Eq. (38) is used in this paper as the convergence condition of recurrence, where $\varepsilon$ is a small number like $1 \times 10^{-3}$ :

$$
\left|\Omega^{(t)}-\Omega^{(t-1)}\right| \leq \varepsilon .
$$




\subsubsection{Intercept parameterization}

In this case, each GenCo takes the first-order derivative of the profit according to $\beta_{k}$. Assuming that $\alpha_{k}=a_{k}$, then the proposed LSF of GenCo $k$ for bidding in the energy market is:

$$
\widehat{\mathrm{MC}}_{k}^{e}=a_{k} q_{k}+\beta_{k}
$$

Taking the derivative of Eq. (33) according to $\beta_{k}$ yields:

$$
\frac{\partial \Omega_{k}}{\partial \beta_{k}}=q_{k} \frac{\partial P^{e}}{\partial \beta_{k}}+\left(P^{e}-a_{k} q_{k}-b_{k}\right) \frac{\partial q_{k}}{\partial \beta_{k}} .
$$

Comparing Eq. (40) with Eqs. (18) and (20) and by some simplification, Relation (41) can be obtained as a recursive equation to iteratively adjust coefficient $\beta_{k}$ of GenCo $k$ :

$$
\beta_{k}^{(t+1)}=b_{k}+\frac{q_{k}^{(t)}}{\sum_{\substack{j=1 \\ j \neq k}}^{G}\left(\frac{1}{a_{j}}\right)}
$$

The intercept of MC can be used as the initial value for $\beta_{k}$, and Relation (38) can be used as stop condition.

\subsection{3. k-parameterization}

In this case, as mentioned before, each GenCo tries to adjust the slope and intercept simultaneously and similarly. The proposed supply function of GenCo $k$ for the energy market is:

$$
\widehat{\mathrm{MC}}_{k}^{e}=k_{k} \cdot\left(a_{k} q_{k}+b_{k}\right)
$$

Deriving Eq. (33) with respect to coefficient $k_{k}$ yields:

$$
\frac{\partial \Omega_{k}}{\partial k_{k}}=q_{k} \frac{\partial P^{e}}{\partial k_{k}}+\left(P^{e}-a_{k} q_{k}-b_{k}\right) \frac{\partial q_{k}}{\partial k_{k}} .
$$

Comparing Eq. (43) to Eqs. (18) and (20) and by some simplification, Eq. (44) can be obtained as a recursive equation to iteratively adjust coefficient $k_{k}$ of GenCo $k$ :

$$
k_{k}^{(t+1)}=1+\left(\frac{q_{k}^{(t)}}{P^{e^{(t)}} \cdot \sum_{\substack{j=1 \\ j \neq k}}^{G} \frac{1}{a_{j} \cdot k_{j}^{(t)}}}\right) \cdot k_{k}^{(t)} .
$$

In this method, we can set $k_{i}^{(0)}=1$ as the initial point. Moreover, we can use Eq. (38) as the condition of recursion stoppage.

\subsection{Energy market with constrained GenCos}

Imagine that there is only one constrained generator in the market (GenCo $i$ ), which is the cheapest. The relationships obtained in the previous subsection can still be used with little changes to choose the optimal bidding strategy. If the power allocated to GenCo $i$ equals its proposed capacity, then the other GenCos have to supply the remained demand. This means that ISO can use $Q_{D}-q_{i}^{\max }$ instead of $Q_{D}$ in the market clearing. Therefore, the bidding of constrained GenCo has no effect on the market settlement price, the power allocated to other GenCos, and their revenues.

By substituting Eq. (16) into Eq. (23) and comparing it with Eq. (18), MCP and the quantity of other non-constrained GenCos are obtained independent of the bidding of constrained GenCo $i$ :

$$
\begin{aligned}
& \lambda_{e}=\frac{Q_{D}-q_{i}^{\max }+\sum_{j=1, j \neq i}^{G}\left(\frac{\beta_{j}}{\alpha_{j}}\right)}{\sum_{j \neq i}^{G}\left(\frac{1}{\alpha_{j}}\right)}, \\
& q_{k}=\frac{Q_{D}-q_{i}^{\max }+\sum_{j=1, j \neq i}^{G}\left(\frac{\beta_{j}-\beta_{k}}{\alpha_{j}}\right)}{\sum_{\substack{j=1 \\
j \neq i}}^{G}\left(\frac{\alpha_{k}}{\alpha_{j}}\right)} \quad(k \neq i) .
\end{aligned}
$$

Therefore, ignoring GenCo $i$ 's bidding parameters, Eqs. (37), (41), and (44) can be modified as follows, where $i$ is the constrained GenCo:

$$
\begin{aligned}
& \alpha_{k}^{(t+1)}=a_{k}+\frac{1}{\sum_{\substack{j=1 \\
j \neq k \\
j \neq i}}^{G}\left(\frac{1}{\alpha_{k}^{(t)}}\right)} \\
& \beta_{k}^{(t+1)}=b_{k}+\frac{q_{k}^{(t)}}{\sum_{\substack{j=1 \\
j \neq k \\
j \neq i}}^{G}\left(\frac{1}{a_{j}}\right)}, \\
& k_{k}^{(t+1)}=1+\left(\frac{q_{k}^{(t)}}{P^{e^{(t)}} \cdot \sum_{\substack{j=1 \\
j \neq k \\
j \neq i}}^{G} \frac{1}{a_{j} \cdot k_{j}^{(t)}}}\right) \cdot k_{k}^{(t)} .
\end{aligned}
$$

\subsection{Integrated market (energy and reserve)}

\subsubsection{Without constrained GenCos}

Imagine that each GenCo is able to bid as linear supply function in both energy and reserve markets. As long as no GenCo is constrained, the formulations of Section 3.1 can be used to choose the optimal bidding strategy for both energy and reserve markets independently. Therefore, the LSF-parameterization 
for participating in the energy market is exactly the relationships in the previous subsection, and the relationships for participating in the reserve market will be:

$$
\begin{aligned}
\gamma_{k}^{(t+1)} & =\gamma_{k}+\frac{1}{\sum_{\substack{j=1 \\
j \neq k}}^{n} \frac{1}{\gamma_{j}^{(t)}}}, \\
\theta_{k}^{(t+1)} & =\theta_{k}+\frac{R_{k}^{(t)}}{\sum_{\substack{j=1 \\
j \neq k}}^{n} \frac{1}{\gamma_{j}}}, \\
k_{k}^{(t+1)} & =1+\left(\frac{R_{k}^{(t)}}{P^{r^{(t)}} \cdot \sum_{\substack{j=1 \\
j \neq k}}^{n} \frac{1}{\gamma_{j} \cdot k_{j}^{(t)}}}\right) \cdot k_{k}^{(t)} .
\end{aligned}
$$

\subsubsection{With constrained GenCos}

In this case, we assume that only GenCo $i$ has encountered the capacity constraint. We can still use the relationship stated in the previous sub-section for LSF parameterizing in both energy and reserve markets, with the difference that we have $\mu_{i} \neq 0$ for the constrained GenCo. Dual variable, $\mu_{i}$, which is the marginal cost of the capacity constraint, shows the relation between these two markets, thereby the players update their tendency toward participating in these two markets.

According to Eqs. (16) and (17), we have:

$$
\lambda_{r}=\left\{\lambda_{e}-\beta_{i}-\alpha_{i} q_{i}\right\}+\theta_{i}+\gamma_{i} R_{i}
$$

The phrase in the brackets in Eq. (53) shows the opportunity cost of supplying the reserve service, instead of producing energy equal to the difference between the MCP and the bidding of GenCo $i$ to the energy market [27]. This means that a constrained GenCo has to decide what extent of its capacity must be allocated to reserve and what extent to the energy market; Eq. (53) shows the opportunity cost of this decision. It also shows that the bidding of a nonconstrained GenCo $\left(\mu_{k}=0\right)$ in any of the markets will not have any effect on its bidding in another market. Therefore, considering $\mu_{i}$ and the relationships stated above, we can determine the GenCo's optimal bidding strategy for participating in the integrated markets of energy and reserve.

\subsection{Elastic loads}

Let us imagine that demand is sensitive to price changes, and its offer is a linear function with a negative slope, but is assumed non-strategic. In this case, ISO aggregates total demands to a linear function as in Eq. (54):

$$
P^{d}=\beta_{0}-\alpha_{0} \cdot Q_{D}
$$

By the similar proof mentioned in Section 2.2, markets clearing price with elastic demand can be determined as in Eq. (55):

$$
P^{e}=\frac{\frac{\beta_{0}}{\alpha_{0}}+\sum_{j=1}^{G}\left(\frac{\beta_{j}}{\alpha_{j}}\right)+\sum_{j=1}^{I}\left(\frac{\mu_{j}}{\alpha_{j}}\right)}{\frac{1}{\alpha_{0}}+\sum_{j=1}^{n}\left(\frac{1}{\alpha_{j}}\right)} .
$$

LSF parameterization for GenCos bidding strategy in the energy market with elastic load will be as follows:

$$
\begin{aligned}
& \alpha_{k}^{(t+1)}=a_{k}+\frac{1}{\frac{1}{\alpha_{0}}+\sum_{\substack{j=1 \\
j \neq k}}^{G}\left(\frac{1}{\alpha_{k}^{(t)}}\right)}, \\
& \beta_{k}^{(t+1)}=b_{k}+\frac{q_{k}^{(t)}}{\frac{1}{\alpha_{0}}+\sum_{\substack{j=1 \\
j \neq k}}^{G}\left(\frac{1}{a_{j}}\right)}, \\
& k_{k}^{(t+1)}=1+\left(\begin{array}{c}
\left.\frac{q_{k}^{(t)}}{P^{(t)}} \times \frac{1}{\frac{1}{\alpha_{0}}+\sum_{\substack{j=1 \\
j \neq k}}^{G} \frac{1}{a_{j} \cdot k_{j}^{(t)}}}\right) \times k_{k}^{(t)} .
\end{array}\right.
\end{aligned}
$$

\subsection{GenCos with multi-generation units}

In this section, it is assumed that every GenCo has multiple generators and bids a LSF for each generation unit. The formulation in the previous sections for $b$-parameterization in the energy market is extended. Based on Eq. (5), the market-clearing price can be rewritten as follows:

$$
P=\frac{Q_{D}+\sum_{i=1}^{G} \sum_{j=1}^{g_{j}}\left(\frac{\beta_{i j}}{\alpha_{i j}}\right)}{\hat{A}},
$$

where:

$$
\hat{A}=\sum_{i=1}^{G} \sum_{j=1}^{g_{j}}\left(\frac{1}{\alpha_{i j}}\right) .
$$

The quantity generated by unit $k$ of GenCo $i$ is calculated as follows:

$$
q_{i k}=\frac{P-\beta_{i k}}{\alpha_{i k}} .
$$


The total profit of GenCo $i$ can be written as follows:

$$
\Omega_{i}=P . \sum_{j=1}^{g_{i}} q_{i j}-\sum_{j=1}^{g_{i}}\left(0.5 a_{i j} \cdot q_{i j}^{2}+b_{i j} \cdot q_{i j}\right) .
$$

Deriving Eq. (62) with respect to $\beta_{i k}$ yields:

$$
\begin{aligned}
\frac{\partial \Omega_{i}}{\partial \beta_{i k}}= & \frac{\partial P}{\partial \beta_{i k}} \cdot q_{i}+\left(P-\mathrm{MC}_{i k}\right) \cdot \frac{\partial q_{i k}}{\partial \beta_{i k}} \\
& +\sum_{\substack{j=1 \\
j \neq k}}^{g_{i}}\left[\left(P-\mathrm{MC}_{i j}\right) \cdot \frac{\partial q_{i j}}{\partial \beta_{i k}}\right] .
\end{aligned}
$$

According to Eq. (60), we have:

$$
\frac{\partial P}{\partial \beta_{i k}}=\frac{1}{\alpha_{i k} \hat{A}}
$$

According to Eq. (61) and considering Eq. (64), we have:

$$
\begin{aligned}
\frac{\partial q_{i k}}{\partial \beta_{i k}} & =\frac{-\hat{A}_{-i k}}{\alpha_{i k} \hat{A}}, \\
\frac{\partial q_{i j}}{\partial \beta_{i k}} & =\frac{1}{\alpha_{i j} \alpha_{i k} \hat{A}}, \quad j \neq k,
\end{aligned}
$$

where:

$$
\hat{A}_{-i k}=\hat{A}-\frac{1}{\alpha_{i j}} .
$$

In intercept parameterization, the slopes of LSF bids are fixed and equal to the marginal cost coefficients; therefore, the term $\left(P-\mathrm{MC}_{i j}\right)$ can be written as follows:

$$
P-\mathrm{MC}_{i j}=\beta_{i k}-b_{i k} .
$$

By considering Eq. (68) and substituting Eqs. (64) to (66) into Eq. (63) and setting it as zero, we have:

$$
-\hat{A} \cdot \beta_{i k}-\sum_{\substack{j=1 \\ j \neq k}}^{g_{i}} \frac{\beta_{i j}}{\alpha_{i j}}=q_{i}+\hat{A}_{-i k} \cdot b_{i k}-\sum_{\substack{j=1 \\ j \neq k}}^{g_{i}} \frac{b_{i j}}{\alpha_{i j}} .
$$

Now, considering that GenCo $i$ has $g_{i}$ units, we have $g_{i}$ equations, such as Eq. (69), that must be solved together:

$$
\left\{\begin{array}{c}
-\hat{A} . \beta_{i 1}-\sum_{\substack{j=1 \\
j \neq 1}}^{g_{i}} \frac{\beta_{i j}}{\alpha_{i j}}=q_{i}+\hat{A}_{-i 1} . b_{i 1}-\sum_{\substack{j=1 \\
j \neq 1}}^{g_{i}} \frac{b_{i j}}{\alpha_{i j}}, \\
\vdots \\
-\hat{A} . \beta_{i g_{i}}-\sum_{j=1}^{g_{i}-1} \frac{\beta_{i j}}{\alpha_{i j}}=q_{i}+\hat{A}_{-i g_{i}} \cdot b_{i g_{i}}-\sum_{j=1}^{g_{i}-1} \frac{b_{i j}}{\alpha_{i j}} .
\end{array}\right.
$$

By solving this system of equation and some simplifications, the relationship for intercept-parameterization is obtained as follows:

$$
\beta_{i k}=b_{i k}+\frac{q_{i}}{\hat{A}_{-i}}
$$

where:

$$
\hat{A}_{-i}=\sum_{l \neq i}^{G} \sum_{j=1}^{g_{j}}\left(\frac{1}{\alpha_{l j}}\right) .
$$

The obtained formula is like Relation (41), the case in which every GenCo has only one generator, with the exception that in Eq. (71), $q_{i}$ is the total quantity generated by all units of GenCo $i$, and $\hat{A}_{-i}$ is calculated excluding all the slopes of GenCo $i$ 's LSF bids.

For elastic loads, as proved in the previous subsection, it can be shown that the formula for interceptparameterization is like Eq. (71) with the exception that $\hat{A}_{-i}$ is calculated as follows:

$$
\hat{A}_{-i}=\frac{1}{\alpha_{0}}+\sum_{l \neq i}^{G} \sum_{j=1}^{g_{j}}\left(\frac{1}{\alpha_{l j}}\right),
$$

where $\alpha_{0}$ is elastic demand price slope.

\section{Simulation results}

To study the different aspects of the proposed method and its accuracy, three test networks utilized in $[15,23,28]$ are used. The analysis will be done in three parts: at first, using the network in [23], the accuracy of the proposed method in choosing the bid for the energy market will be investigated, and it will be shown that the proposed method is accurate similar to metaheuristic approaches, yet it is faster in solving bidding strategy problems in pool-based power markets. Then, using the network [28], application of the proposed method for multi-generation unit case is considered, and the capability of the proposed method is proven. Finally, using the network like [15], application of the proposed method for choosing the bid in the integrated energy and SR market will be investigated.

\subsection{Energy market-single-generator case}

In [23], using a Co-evolutionary Genetic Algorithm (CGA) for determining the market equilibrium, the linear supply function bidding parameters to participate in the energy market based on different competition models have been adjusted. The data of GenCos' cost function are represented in Table $1 . \quad$ Imagine that demand has non-zero elasticity and the aggregated demand parameters are $\alpha_{0}=10 \$ /($ MWh.GWh) and $\beta_{0}=350 \$ / \mathrm{MWh}$. In this part, the GenCos' bidding parameters will be adjusted using the proposed method and then compared with the study results of Zhang et al. in [23]. 
Table 1. Cost coefficients of the five generators.

\begin{tabular}{ccc}
\hline GenCo & $\boldsymbol{a}_{\boldsymbol{i}} \$ \mathbf{( M W h . G W h )}$ & $\boldsymbol{b}_{\boldsymbol{i}}(\$ / \mathbf{M W h})$ \\
\hline$G_{1}$ & 2.684 & 12 \\
$G_{2}$ & 4.615 & 12 \\
$G_{3}$ & 1.789 & 8 \\
$G_{4}$ & 1.930 & 8 \\
$G_{5}$ & 4.615 & 12 \\
\hline
\end{tabular}

Table 2. Result of the market with perfect competition.

\begin{tabular}{cccc}
\hline GenCo & MCP & $\boldsymbol{q}_{\boldsymbol{i}}$ & Profit \\
\hline$G_{1}$ & & 5.5359 & 41.1729 \\
$G_{2}$ & & 3.2232 & 23.9722 \\
$G_{3}$ & 26.87 & 10.5505 & 99.5703 \\
$G_{4}$ & & 9.7797 & 92.2960 \\
$G_{5}$ & & 3.2232 & 23.9722 \\
\hline
\end{tabular}

\subsubsection{Competitive energy market}

It is supposed that the market is completely competitive, and each GenCo bids its marginal cost in the energy market. According to Eqs. (23) to (25), the market equilibrium with perfect competition for these five Gen Co systems is shown in Table 2. According to the data of Table $1, G_{2}$ and $G_{5}$ are the most expensive producers and $G_{3}$ is the cheapest. Therefore, ISO gives the highest share of production to $G_{3}$ and the least to $G_{2}$ and $G_{5}$ in order to increase the social welfare. Since the market participants are paid uniform, the cheapest GenCo, i.e. $G_{3}$, obtains the highest profit and the expensive GenCos, i.e. $G_{2}$ and $G_{5}$, obtain the lowest profit.

\subsubsection{Oligopoly energy market}

In this case, it is supposed that all five GenCos are strategic and bid strategically in an oligopoly energy market; so, they try to maximize their expected profit by deviating the market price from its competitive value. To study the effects of GenCos' decisions on market settlement, all the three competition models, i.e. $a-, b$-, and $k$-parameterizations, should be investigated. Considering that the capacity of the GenCos is large enough, the results of LSF-parameterizations based on the proposed relationships in Section 3.4 are shown in Table 3.

Figure 2 shows the convergences of supply function parameters. According to the similarity of the marginal cost parameters of $G_{2}$ and $G_{5}$, the same diagrams are drawn for both.

\subsubsection{Discussing the results}

According to the results shown in Tables 1 to 3, we can conclude as following:

1. It can be seen from Table 3 that the proposed method leads to the same results shown in [23] for
Table 3. Results of the oligopolistic energy market.

\begin{tabular}{cccccc}
\hline $\begin{array}{c}\text { Strategic } \\
\text { variable }\end{array}$ & GenCo & Optimal & $\boldsymbol{P}^{\boldsymbol{e}}$ & $\boldsymbol{q}_{\boldsymbol{i}}$ & $\boldsymbol{\Omega}_{\boldsymbol{i}}$ \\
\hline \multirow{2}{*}{$\alpha_{i}$} & $G_{1}$ & 3.517 & & 5.92 & 76.3167 \\
& $G_{2}$ & 5.386 & & 3.87 & 46.1131 \\
& $G_{3}$ & 2.689 & 32.845 & 9.24 & 153.1792 \\
& $G_{4}$ & 2.817 & & 8.82 & 144.0601 \\
& $G_{5}$ & 5.3863 & & 3.87 & 46.1131 \\
& & & & & \\
$\beta_{i}$ & $G_{1}$ & 15.660 & & 5.89 & 68.2708 \\
& $G_{2}$ & 14.131 & & 3.76 & 40.7090 \\
& $G_{3}$ & 14.626 & 31.5009 & 9.43 & 142.0886 \\
& $G_{4}$ & 14.142 & & 8.99 & 133.3096 \\
& $G_{5}$ & 14.131 & & 3.76 & 40.7090 \\
& & & & & \\
& $G_{1}$ & 1.1594 & & 5.90 & 73.0111 \\
& $G_{2}$ & 1.089 & & 3.82 & 43.8930 \\
$k_{i}$ & $G_{3}$ & 1.309 & 32.3001 & 9.32 & 148.7884 \\
& $G_{4}$ & 1.284 & & 8.89 & 139.7811 \\
& $G_{5}$ & 1.089 & & 3.82 & 43.8930 \\
\hline & & & & &
\end{tabular}

all three competition models of LSF parameterization. As explained in introduction section, one of the specific characteristics of the proposed method is its simplicity. In [23], the game is supposed to be with complete information and the market equilibrium is calculated by using CGA, but it can be easily determined using our proposed iterationbased equations, and having these equations, we do not need to use optimizing tools;

2. There are several techniques for evaluating the performance of optimization algorithms. In cases where meta-heuristic optimization algorithms, such as the genetic algorithm, have to be evaluated, the most common performance metric is the total Number of objective Function Evaluations (NFE). Other than that, another metric is the time needed for completing the task, but that is directly proportional to the NFE metric. The better approach has the lower NFE and time. NFE for the proposed method is equal to the number of iterations, i.e., 10; but, for GA, NFE is equal to 5470 . The time of simulation is 6.7 seconds for GA and 0.023 seconds for the proposed method. Therefore, these two important criteria confirm that the proposed method can be faster;

3. In simulating program, Relation (38) is used as the stoppage condition in which $\varepsilon$ equals $1 \times 10^{-3}$. In reality, this measure is too small for profit. The number of iterations needed for convergence of all the three models of parameterization with 


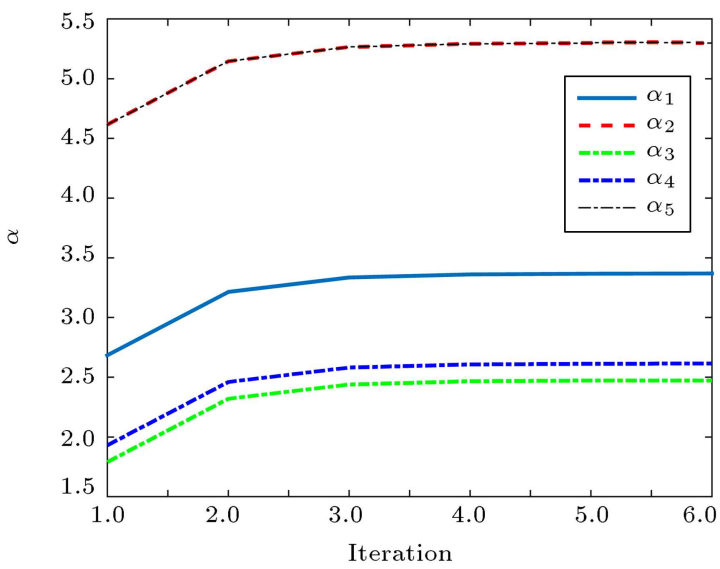

(a) Setting $\alpha_{i}$

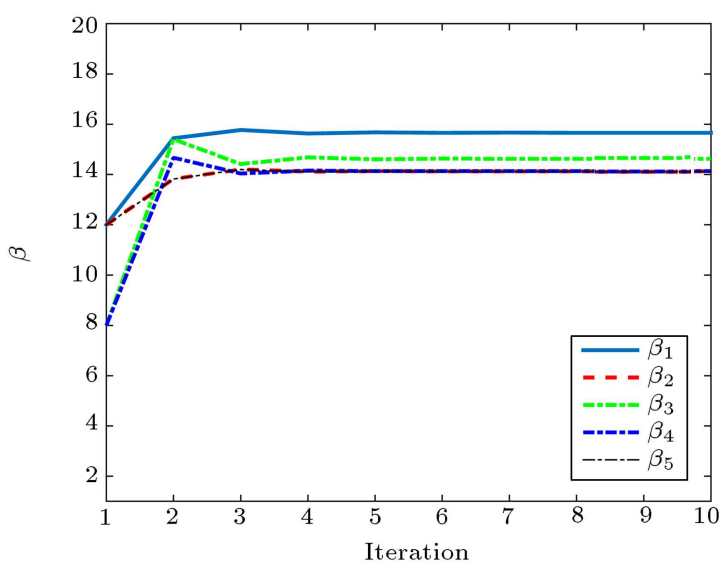

(b) Setting $\beta_{i}$

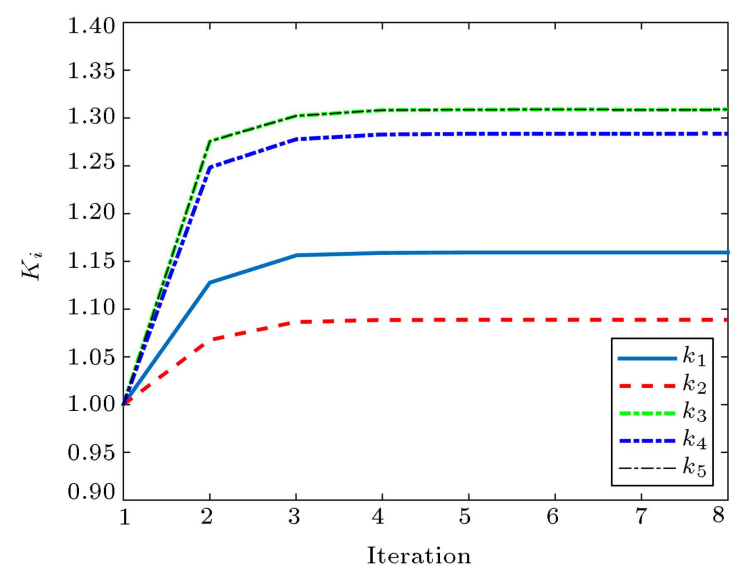

(c) Setting $k_{i}$

Figure 2. Convergence of the supply function parameters.

different values for $\varepsilon$ is shown in Table 4 . It can be seen that with bigger numbers for $\varepsilon$, the algorithm is converged with less iteration. In [29], the negotiation for settling the market is explained. Therefore, for a market with negotiated settling mechanism, the proposed method can be profitable for Gen Co due to convergence with less iteration;

4. By comparing Tables 2 and 3, it can be seen that the MCP in all incomplete competition mod-
Table 4. Number of iterations for convergence of algorithm.

\begin{tabular}{ccccc}
\hline Strategic & \multicolumn{4}{c}{ Number of iterations } \\
\cline { 2 - 5 } variable & $\boldsymbol{\varepsilon}=\mathbf{1 0}^{-\mathbf{3}}$ & $\boldsymbol{\varepsilon}=\mathbf{1 0}^{-\mathbf{2}}$ & $\boldsymbol{\varepsilon}=\mathbf{1 0}^{-\mathbf{1}}$ & $\boldsymbol{\varepsilon}=\mathbf{1 0}^{\mathbf{0}}$ \\
\hline$\alpha_{i}$ & 10 & 8 & 6 & 5 \\
$\beta_{i}$ & 10 & 8 & 6 & 4 \\
$k_{i}$ & 8 & 7 & 6 & 4 \\
\hline
\end{tabular}

els $\left(a^{-}, b-\right.$, or $k$-parameterization $)$ is more than that in complete competition. The MCP in $a$ parameterization is more than those in the two other models, and in b-parameterization, it is less than those in the two others. In addition, since the demand is supposed to be elastic, it reacts to this price increase, and with this increase, the demand will reduce. In fact, in complete competition case, the demand is the highest $\left(Q_{D}=32.31 \mathrm{MW}\right)$, but in b-parameterization, demand is the lowest $\left(Q_{D}=\right.$ 31.71 MW). Thus, in the case of a-parameterization, the highest price deviation and capacity withholding is done. On the contrary, it can be seen that competitive model for $k$-parameterization shows more moderate results compared with the two other models. Therefore, like what was explained in [27], $k$-parameterization leads to results closer to the real markets.

\subsection{Energy market-multi-generator case}

In this case, we study a network with three GenCos in which every GenCo has more than one unit. Table 5 shows generators' cost function parameters, which are like the network studied in [28]. Total demand is assumed to be $1000 \mathrm{MW}$.

Table 6 shows the market simulation results under complete competition condition where every GenCo bids its marginal price for each of its units to ISO, and Table 7 shows the market simulation under incomplete competition condition. It is assumed that the GenCos are allowed to adjust only the intercept ( $b$ parameterization) using Eq. (71). The comparison of the results of Tables 6 with 7 shows that because of tacit collusion, each Gen Co can increase the LSF intercept. This increasing trend continues until convergence condition, like Eq. (38), is satisfied.

For verification of the proposed method, assume that each GenCo has only one generator, i.e. the six generators in Table 5 are six GenCos and they adjust

Table 5. Coefficients of the generators' cost functions.

\begin{tabular}{cccccc}
\hline \multirow{2}{*}{ GenCo } & \multicolumn{2}{c}{ Unit $\mathbf{1}$} & & \multicolumn{2}{c}{ Unit 2} \\
\cline { 2 - 3 } \cline { 5 - 6 } & $\boldsymbol{a}_{\boldsymbol{i} \mathbf{1}}$ & $\boldsymbol{b}_{\boldsymbol{i} \mathbf{1}}$ & & $\boldsymbol{a}_{\boldsymbol{i} \mathbf{2}}$ & $\boldsymbol{b}_{\boldsymbol{i} \mathbf{2}}$ \\
\hline$G_{1}$ & 0.04 & 10 & & 0.06 & 15 \\
$G_{2}$ & 0.08 & 20 & & 0.05 & 12 \\
$G_{3}$ & 0.06 & 16 & & 0.09 & 19 \\
\hline
\end{tabular}


A. Azadi Hematabadi and A. Akbari Foroud/Scientia Iranica, Transactions D: Computer Science \& ... 24 (2017) 3231-32463241

Table 6. Market simulation results under perfect competition.

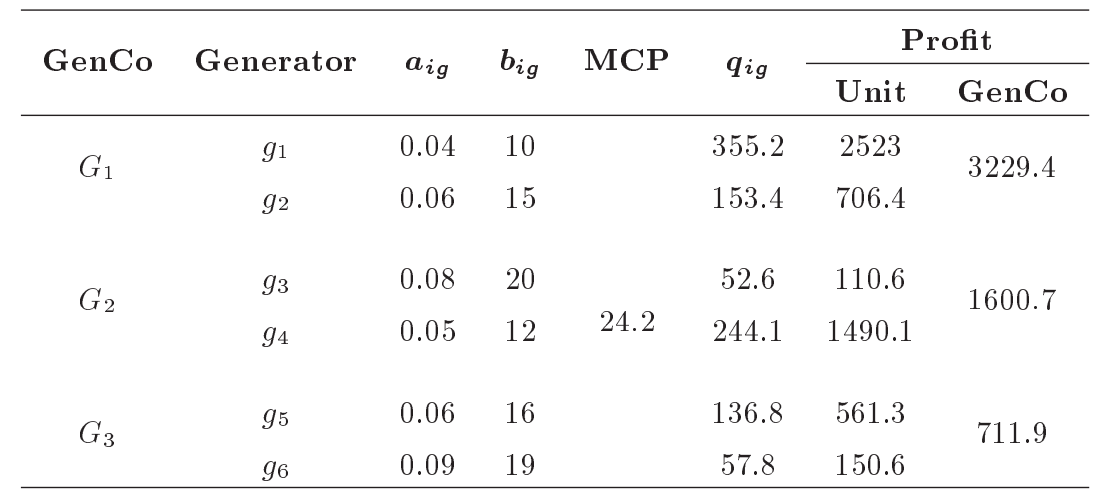

Table 7. Market simulation results under imperfect competition.

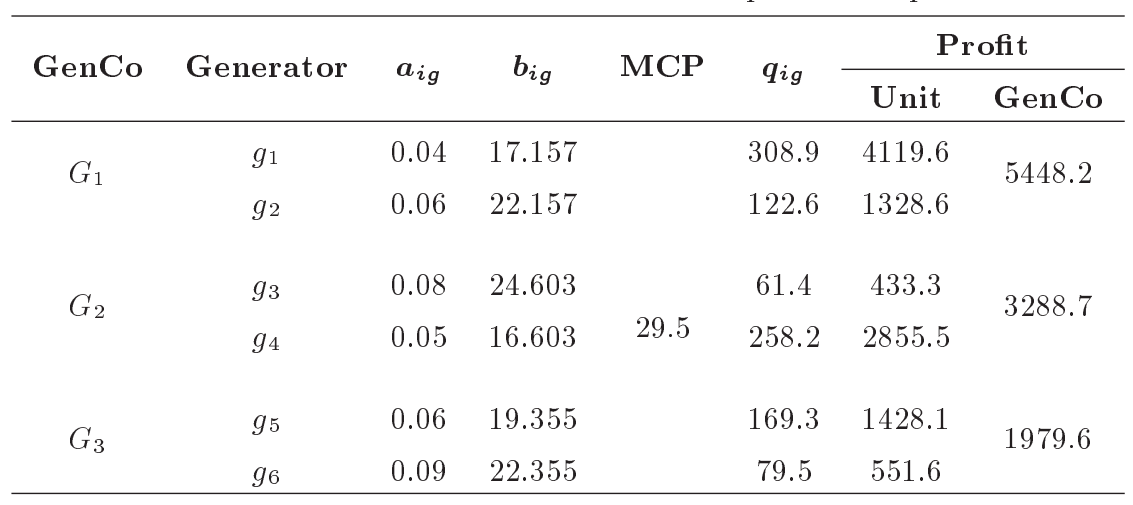

Table 8. Market simulation results with incomplete competition-single-generator case.

\begin{tabular}{|c|c|c|c|c|c|c|}
\hline \multirow{2}{*}{ GenCo } & \multirow{2}{*}{$a_{i g}$} & \multirow{2}{*}{$b_{i g}$} & \multirow{2}{*}{ MCP } & \multirow{2}{*}{$q_{i g}$} & \multicolumn{2}{|c|}{ Profit } \\
\hline & & & & & Unit & GenCo \\
\hline$G_{1}$ & 0.04 & 14.04 & & 312.4 & 3213 & \multirow{2}{*}{4292} \\
\hline$G_{2}$ & 0.06 & 16.89 & & 160.7 & 1078 & \\
\hline$G_{3}$ & 0.08 & 20.8 & \multirow[b]{2}{*}{26.53} & 71.7 & 263 & \multirow{2}{*}{2294} \\
\hline$G_{4}$ & 0.05 & 14.85 & & 233.7 & 2031 & \\
\hline$G_{5}$ & 0.06 & 17.72 & & 146.9 & 900 & \multirow{2}{*}{1211} \\
\hline$G_{6}$ & 0.09 & 19.82 & & 74.6 & 311 & \\
\hline
\end{tabular}

their strategic parameters based on Eq. (41). The market simulation results are shown in Table 8 . The comparison of the results of Table 6 with those of Table 8 shows that the profit of each generator in a multi-generator case is greater than its profit in a single-generator case, with bigger adjusted intercept parameters. For example, if generators $g_{1}$ and $g_{2}$ form GenCo $1\left(G_{1}\right)$ and bid with respect to each other in the multi-generator case, they would gain 4119.6 and $1328.6 \$$, respectively, and the total profit of $G_{1}$ would be $5448.2 \$$, as shown in Table 7 ; but, in the single-generator case, $g_{1}$ and $g_{2}$ gain 3213 and $1078 \$$, respectively. Assuming that they form $G_{1}$ as a GenCo, the total profit of $G_{1}$ would be $4292 \$$ that is less than the total profit of multi-generator case. It can be said that the multi-generator case is the same as explicit collusion case where some generators coalesce into a GenCo.

\subsection{Integrated energy and spinning reserve}

In this part, the behavior of GenCos for participating in the integrated energy and spinning reserve market using the proposed method is studied. The GenCos' energy and reserve cost function parameters according to data in [15] and with a little change are shown in Table 9. It is supposed that the demand is non-elastic and equals $1500 \mathrm{MW}$.

As a result, according to the explanations in Section 2.2, the SR requirement for the network will be $150 \mathrm{MW}$. To study the proposed method, five cases in two parts are considered, in which the completely

Table 9. Coefficients of the energy and reserve cost functions.

\begin{tabular}{cccccc}
\hline GenCo & \multicolumn{2}{c}{$\begin{array}{c}\text { Reserve cost } \\
\text { function } \\
\text { coefficients }\end{array}$} & & \multicolumn{2}{c}{$\begin{array}{c}\text { Energy cost } \\
\text { function } \\
\text { coefficients }\end{array}$} \\
\cline { 2 - 3 } \cline { 5 - 6 } & $\boldsymbol{\gamma}_{\boldsymbol{i}}$ & $\boldsymbol{\theta}_{\boldsymbol{i}}$ & & $\boldsymbol{a}_{\boldsymbol{i}}$ & $\boldsymbol{b}_{\boldsymbol{i}}$ \\
\hline$G_{1}$ & 0.08 & 4 & & 0.05 & 10 \\
$G_{2}$ & 0.1 & 5 & & 0.08 & 15 \\
$G_{3}$ & 0.1 & 7 & & 0.06 & 20 \\
\hline
\end{tabular}


competitive market and oligopoly market with three players are studied, respectively.

\subsubsection{Perfect competition market}

In this section, the market is assumed to be perfect competitive. Market without constrained GenCo and Market with constrained GenCo are analyzed in Cases $\mathrm{A}$ and $\mathrm{B}$, respectively.

- Case (A) Without any constrained GenCo: In this case, the market is assumed completely competitive; therefore, all GenCos bid their marginal cost to the ISO and none of them manipulates its offer to maximize its revenue. It is also supposed that none of the GenCos faces capacity constraint, and the total production and spinning reserve for each GenCo is less than its total capacity. The results of market settlement under these conditions are shown in the first row of Table 10. Coefficient $\mu_{i}$ for all units equals zero because none of them is constrained. Each GenCo increases its generation up to a point where its marginal cost equals market price. As a result, cheaper Gen Cos (like $G_{1}$ ) have more generation in the market;

- Case (B) $G_{1}$ is constrained with maximum capacity of $630 \mathrm{MW}$ : In this case, it is supposed that total capacity of cheaper GenCo, i.e. $G_{1}$, equals $630 \mathrm{MW}$. By limiting $G_{1}$, the simulation results will be in accordance to row 2 in Table 10. It can be seen that because $G_{1}$ is constrained, its coefficient $\mu_{1}$ will be non-zero, but for $G_{2}$ and $G_{3}$, it equals zero because none of them is constrained. By comparing rows 1 and 2 from Table 10, it can be seen that the Gen Cos profit in Case B is more than that in Case A, which is due to fixed load and constraining GenCo $G_{1}$. More capacity is allocated to the GenCos that are more expensive; as a result, the market price in both energy and reserve markets will increase, leading to an increase in GenCos' profit. The price markups for energy and reserve markets are 14.1 and 5.1, respectively.

In Case A, where none of the GenCos is constrained, ISO allocates most demands and SR requirements to the cheaper GenCo $G_{1}$, up to a point that its bidding is not higher than that of the others. While in Case B, where $G_{1}$, the cheaper GenCo, is bounded, it can be seen that the dispatch by ISO is the way that most of the capacity of cheaper and constrained GenCo $G_{1}$ is allocated to the energy market and the rest of its capacity is allocated to supplying the SR services. This is due to more quantity and price of the energy market with respect to the SR market.

\subsubsection{Oligopoly integrated market}

In this part, each GenCo plays as a price-maker and tries to set its bids by simulation of its competitors' actions logically and in accordance with relationships in Section 3. It is also supposed that each GenCo maximizes its profit adjusting the LSF parameters in both integrated energy and reserve markets.

Table 10. Results of the oligopolistic integrated market.

\begin{tabular}{|c|c|c|c|c|c|c|c|c|c|c|c|}
\hline Case & GenCo & $\alpha_{i}$ & $\boldsymbol{\beta}_{i}$ & $\gamma_{i}$ & $\theta_{i}$ & $\mu_{i}$ & $P^{e}$ & $\boldsymbol{P}^{r}$ & $q_{i}$ & $\boldsymbol{R}_{i}$ & $\Omega\left(\times 10^{3}\right)$ \\
\hline \multirow{3}{*}{ A } & $G_{1}$ & 0.05 & 10 & 0.08 & 4 & 0 & \multirow{3}{*}{34.1} & \multirow{3}{*}{7.6} & 703 & 73 & 12.6 \\
\hline & $G_{2}$ & 0.08 & 15 & 0.1 & 5 & 0 & & & 377 & 48 & 5.8 \\
\hline & $G_{3}$ & 0.06 & 20 & 0.1 & 7 & 0 & & & 410 & 28 & 5.3 \\
\hline \multirow{3}{*}{ B } & $G_{1}$ & 0.05 & 10 & 0.08 & 4 & 7.5 & \multirow{3}{*}{48.2} & \multirow{3}{*}{12.7} & 614 & 16 & 14.2 \\
\hline & $G_{2}$ & 0.08 & 15 & 0.1 & 5 & 0 & & & 415 & 77 & 7.2 \\
\hline & $G_{3}$ & 0.06 & 20 & 0.1 & 7 & 0 & & & 470 & 57 & 6.8 \\
\hline \multirow{3}{*}{$\mathrm{C}$} & $G_{1}$ & 0.05 & 27.13 & 0.08 & 6.64 & 0 & \multirow{3}{*}{52.1} & \multirow{3}{*}{10.8} & 500 & 53 & 15.1 \\
\hline & $G_{2}$ & 0.08 & 15 & 0.1 & 5 & 0 & & & 464 & 59 & 8.8 \\
\hline & $G_{3}$ & 0.06 & 20 & 0.1 & 7 & 0 & & & 536 & 38 & 8.7 \\
\hline \multirow{3}{*}{$\mathrm{D}$} & $G_{1}$ & 0.05 & 10 & 0.08 & 5.16 & 8.1 & \multirow{3}{*}{48.5} & \multirow{3}{*}{15.1} & 607 & 23 & 14.4 \\
\hline & $G_{2}$ & 0.08 & 15 & 0.1 & 8.12 & 0 & & & 418 & 70 & 7.5 \\
\hline & $G_{3}$ & 0.06 & 20 & 0.1 & 9.51 & 0 & & & 475 & 57 & 7.1 \\
\hline \multirow{3}{*}{$\mathrm{E}$} & $G_{1}$ & 0.05 & 29.97 & 0.08 & 6.38 & 3.7 & \multirow{3}{*}{62.8} & \multirow{3}{*}{13.9} & 582 & 48 & 22.7 \\
\hline & $G_{2}$ & 0.08 & 27.16 & 0.1 & 8.43 & 0 & & & 446 & 55 & 13.7 \\
\hline & $G_{3}$ & 0.06 & 34.51 & 0.1 & 9.12 & 0 & & & 472 & 47 & 13.7 \\
\hline
\end{tabular}


- Case (C) Integrated market with a strategic GenCo: In this case, suppose that $G_{1}$ is the only strategic GenCo in the market which tries to obtain the highest profit by participating in energy and reserve markets using LSF parameterization. None of the GenCos is constrained and the production of all the GenCos is less than their capacity. The market equilibrium is shown in row 3 of Table 10. By comparing rows 1 and 3 in Tables 10, it can be seen that $G_{1}$ tries to maximize its profit by withholding its capacity through bidding more than competitive level in both energy and reserve markets. The total capacity of $G_{1}$ decreases from $779 \mathrm{MW}$ in Case A to $553 \mathrm{MW}$ in Case $\mathrm{C}$, and the most decrease is in the capacity given to the energy market and, as a result, leads to the energy market price deviation from $34 \$ / \mathrm{MW}$ to $52 \$ / \mathrm{MW}$. $G_{1}$ profit increases from $12.6 \times 10^{3} \$$ in Case A to $15.1 \times 10^{3} \$$ in Case C. Also, it can be seen that although the bidding of the price taker GenCos, i.e., $G_{2}$ and $G_{3}$, remains fixed as their marginal cost, their profit increases because of $G_{1}$ strategic bidding. This is one of the characteristics of uniform settlement mechanism.

- Case (D) Competing in reserve market: In this case, all GenCos are allowed to parameterize the supply function in reserve market, and the energy market is considered competitive. The capacity of $G_{1}$ is supposed to be $630 \mathrm{MW}$. The market equilibrium under this situation is shown in the fourth row of Table 10. It can be seen from the results that the players manipulate their supply function's coefficients in reserve market and increase the reserve price higher than the competitive level (i.e., Case A). As a result, the GenCos' revenue increases despite the lower production.

By comparing the two Cases of B and D, it can be seen that according to the opportunity cost between the two markets, GenCo $G_{1}$ allocates $7 \mathrm{MW}$ of its capacity to reserve; therefore, despite the game in reserve market, this Gen Co induces a small increase in the market price. Since demand is not elastic, the reserve requirement is fixed. Therefore, if cheaper GenCo $G_{1}$ decides to supply less reserve, other more expensive GenCos will compensate for this decrease, and thus, the price of spinning reserve services increases. This increase, caused by exercising GenCos' market power, brings them more profit and, therefore, decreases social welfare.

- Case (E) Competition in both energy and SR markets: In this case, we assume that all three GenCos behave strategically and compete with each other in both energy and SR markets; $G_{1}$ has the total capacity of $630 \mathrm{MW}$. The result of integrated market equilibrium is shown in row five of Table 10 . By comparing the rows of Table 10, it can be seen that

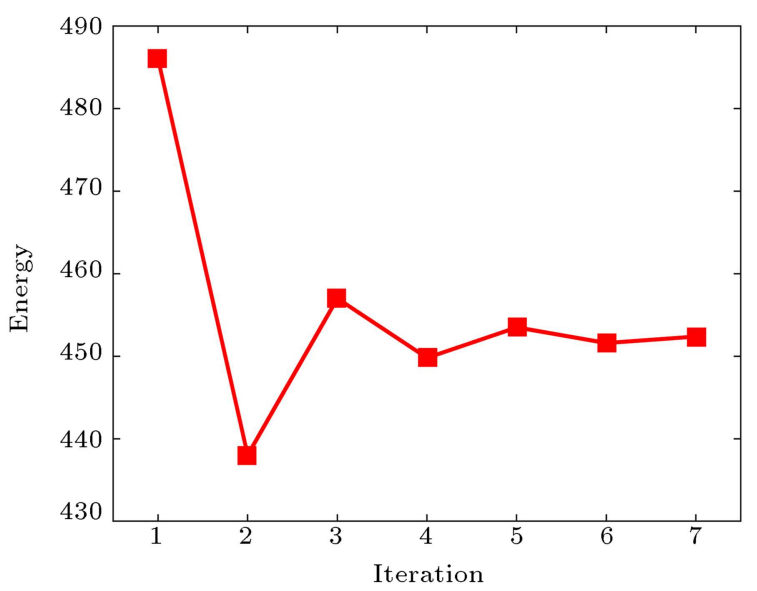

(a) Reserve capacity

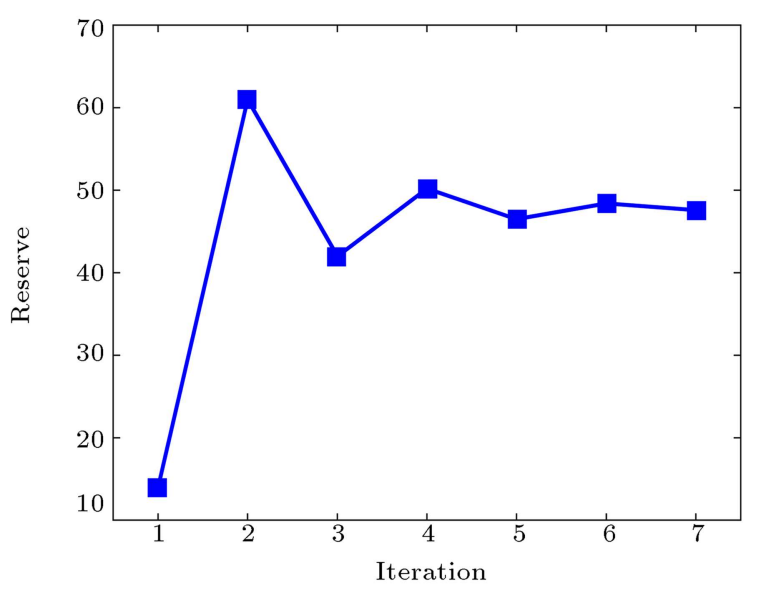

(b) Energy quantity

Figure 3. The iterative gaming of $G_{1}$ between energy and reserve markets.

the simultaneous game in both markets results in more payoff than in the previous cases. Figure 3 shows the gaming of $G_{1}$ for shifting the capacity between these two markets and choosing the best option in an iterative process. Through this game, $G_{1}$ learns that the optimal strategy is to decrease $32 \mathrm{MW}$ of its energy capacity (Case B) and allocates this to SR capacity; therefore, the energy market price increases from $48.2 \$ / \mathrm{MWh}$ to $62.82 \$ / \mathrm{MWh}$. Since market clearings are done simultaneously, this decrease in the energy market leads to the increase in supplying the reserve services, and ISO allocates $48 \mathrm{MW}$ of the total reserve requirement to $G_{1}$ than $16 \mathrm{MW}$ in Case B. That is the reason why despite the manipulation of the supply function parameters, because of the effective presence of the cheaper GenCo $G_{1}$, the SR price has not had a noticeable increase just like the energy market. What is important for every GenCo is to get more profit from both markets of energy and reserve, and all the three GenCos have reached this goal by parameterizing their supply function in this integrated market. 


\section{Conclusions}

In this paper, a new solution method of suppliers bidding strategy is proposed. The proposed method is based on iterative process and developed for both elastic and non-elastic demands. it is efficient for participating in energy markets and integrated energy and SR markets. The proposed method was also developed for multi-generator cases in which every GenCo has more than one generator (generation unit). The gaming of the producer for allocating the capacity between energy and reserve markets and the opportunity cost between them are also considered in the proposed method.

The correctitude of the proposed method and its efficiency was investigated by three case studies. At first, the accuracy of the proposed bidding strategy for participating in the energy market with five GenCos test cases and elastic demand was considered, which was studied by Zhang et al. who had adjusted the LSF parameters using CGA. The results of LSF parameterization by the iterative proposed method for all the three models of competitions, i.e. $a-, b$-, and $k$-parameterization, show that the proposed method is accurate and effective, and the results are the same as those shown in the mentioned research through CGA. Some of the advantages of the proposed method can be highlighted as follows:

- Simplicity compared with other solution tools such as GA;

- Immunity to the problems of convergence;

- High convergence speed with low iteration number.

However, the proposed method can be combined with metaheuristic algorithms, so that a modified optimization algorithm with a fast technic for calculating the equilibrium point of the market can be introduced in future studies.

Then, a network with three GenCos, each with two generators, has been used for investigating the capability of the proposed method in producers' bidding strategy for multi-generator GenCo case. The results show that each GenCo achieves more profit than the complete competition. In addition, it is shown that each generator can achieve more profit than the singlegenerator case in which each generator is a GenCo and there is no coalification among the generators. It can be said that the multi-generator case is the same as explicit collusion case where some generators coalesce into a GenCo.

Finally, a network with three GenCos has been used for investigating the integrated energy and SR markets by the proposed method. In this case, it was shown that the proposed method is effective in participating in the markets with both constrained and non-constrained GenCos. It was also shown that the constrained GenCo learns during an iterative process how much of its overall capacity should be allocated to the energy market to maximize its expected profit in the integrated market.

\begin{tabular}{|c|c|}
\hline \multicolumn{2}{|c|}{ Nomenclature } \\
\hline$J$ & Index for GenCos \\
\hline$I$ & Index for constrained GenCos \\
\hline$K$ & Index for unconstrained GenCos \\
\hline$G$ & Set of GenCos \\
\hline$g_{i}$ & $\begin{array}{l}\text { The number of generation units of } \\
\text { GenCo } i\end{array}$ \\
\hline$I$ & Set of constrained GenCos \\
\hline$C_{i k}^{e}$ & $\begin{array}{l}\text { Cost function of energy generation for } \\
\text { unit } k \text { of GenCo } i\end{array}$ \\
\hline$C_{i k}^{r}$ & $\begin{array}{l}\text { Cost function of SR for unit } k \text { of } \\
\text { GenCo } i\end{array}$ \\
\hline$a_{j}, b_{j}$ & $\begin{array}{l}\text { Energy cost function coefficients of } \\
\text { GenCo } j\end{array}$ \\
\hline$\rho_{j}, v_{j}$ & $\begin{array}{l}\text { Spinning reserve cost function } \\
\text { coefficients of GenCo } j\end{array}$ \\
\hline$\alpha_{0}$ & Price slope for elastic demand curve \\
\hline$\beta_{0}$ & Price intercept for demand curve \\
\hline$Q_{D}$ & Total demand \\
\hline$Q_{S R}$ & Total required spinning reserve \\
\hline$q_{i}^{\max }$ & Maximum capacity of GenCo $i$ \\
\hline$P^{e}$ & Energy market price \\
\hline$P^{r}$ & SR market price \\
\hline$\alpha_{j}$ & $\begin{array}{l}\text { Slope of the supply function for GenCo } \\
j\end{array}$ \\
\hline$\beta_{j}$ & $\begin{array}{l}\text { Intercept of the supply function for } \\
\text { GenCo } j\end{array}$ \\
\hline$q_{j}$ & Power supplied by GenCo $j$ \\
\hline$R_{j}$ & SR supplied by GenCo $j$ \\
\hline
\end{tabular}

\section{References}

1. Ma, X., Wen, F., Ni, Y. and Liu, J. "Towards the development of risk-constrained optimal bidding strategies for generation companies in electricity markets", Electric Power System Research, 73(3), pp. 305312 (2005).

2. Azadeh, A., Ghaderi, S., Pourvalikhan Nokhandan, B. and Sheikhalishahi, M. "A new genetic algorithm approach for optimizing bidding strategy viewpoint of profit maximization of a generation company", Expert Systems with Applications, 39(1), pp. 15651574 (2012).

3. Niknam, T., Sharifinia, S. and AzizipanahAbarghooee, R. "A new enhanced bat-inspired algorithm for finding linear supply function 
equilibrium of GENCOs in the competitive electricity market", Energy Conversion and Management, 76, pp. 1015-1028 (2013).

4. Shafie-khah, M., Parsa Moghaddam, M. and SheikhEl-Eslami, M.K. "Development of a virtual power market model to investigate strategic and collusive behavior of market players", Energy Policy, 61(C), pp. 717-728 (2013).

5. Klemperer, P. and Meyer, M. "Supply function equilibria in oligopoly under uncertainty", Econometrica, 57(6), pp. 1243-1277 (1989).

6. Green, R. "Increasing competition in the British electricity spot market", The Journal of Industrial Economics, 44(2), pp. 205-216 (1996).

7. Baldick, R. "Electricity market equilibrium models: the effect of parameterization", IEEE Transaction on Power System, 17(4), pp. 1170-1176 (2002).

8. Petoussis, A.G., Zhang, X., Petoussis, S.G. and Godfrey, K.R. "Parameterization of linear supply functions in nonlinear AC electricity market equilibrium modelspart I: Literature review and equilibrium algorithm", IEEE Transaction on Power System, 28(2), pp. 650658 (2013).

9. Petoussis, A.G., Petoussis, S.G., Zhang, X.P. and Godfrey, K.R. "Market equilibrium in congested transmission networks with transformer tap-ratio control", Proc. IEEE PES Power Tech Conf. Lausanne, Switzerland July, pp. 949-954 (2007).

10. Niknam, T., Azizipanah-Abarghooee, R. and Aghaei, J. "A new modified teaching learning algorithm for reserve constrained dynamic economic dispatch", IEEE Transaction on Power System, 28, pp. 749-63 (2013).

11. Lashkar Ara, A., Aghaei, J., Alaleh, M. and Barati, H. "Contingency-based optimal placement of Optimal Unified Power Flow Controller (OUPFC) in electrical energy transmission systems", Scientia Iranica, Transactions D, 20(3), pp. 778-785 (2013).

12. Javadi, M.S., Esmaeel Nezhadb, A. and Sabramoozc, S. "Economic heat and power dispatch in modern power system harmony search algorithm versus analytical solution", Scientia Iranica, Transactions D, 19(6), pp. 1820-1828 (2012).

13. Eslami-nia, M., Mohammadian, M. and Hemmatpour, M.H. "An approach for increasing wind power penetration in deregulated power system", Scientia Iranica, Transactions $D$.

14. Kirschen, D.S. and Strbac, G., Fundamentals of Power System Economics, Chichester, West Sussex, England, John Wiley \& Sons (2004).

15. Bautista, G., Quintana, V.H. and Aguado, J.A. "An oligopolistic model of an integrated market for energy and spinning reserve", IEEE Transaction on Power System, 21(1), pp. 132-142 (2006).

16. De la Torre, S., Conejo, A.J. and Contreras, J. "Simulating oligopolistic pool-based electricity markets: A multi-period approach", IEEE Transaction on Power System, 18(4), pp. 1547-1555 (2003).

17. Haghighat, H., Seifi, H. and Kian, A.R. "Gaming analysis in joint energy and spinning reserve markets", IEEE Transaction on Power System, 22(4), pp. 20742085 (2007).

18. Gonzalez, P., Villar, J., Diaz, C.A. and Campos, F.A. "Joint energy and reserve markets: Current implementations and modeling trends", Electric Power System Research, 109, pp. 101-111 (2014).

19. Reddy, S.S., Bijwe, P.R. and Abhynkar, A.R. "Joint energy and spinning reserve market clearing incorporating wind power and load forecast uncertainties", IEEE System Journal, 9(1), pp. 152-164 (2013).

20. Nabavia, S.M.H., Kazemia, A. and Masoum, M.A.S. "Social welfare maximization with fuzzy based genetic algorithm by TCSC and SSSC in double-sided auction market", Scientia Iranica, Transactions D, 19(3), pp. 745-758 (2012).

21. Gountis, V. and Bakirtzis, A. "Bidding strategies for electricity producers in a competitive electricity marketplace", IEEE Transaction on Power System, 19(1), pp. 356-365 (2004).

22. Naghibi-Sistani, M.B., Akbarzadeh-Tootoonchi, M.R., Javidi-Dashte-Bayaz, M.H. and Rajabi-Mashhadi, H. "Application of Q-learning with temperature variation for bidding strategies in market based power systems", Energy Conversion and Management, 47, pp. 15291538 (2006).

23. Zhang, S., Chung, C., Wong, K. and Chen, H. "Analyzing two-settlement electricity market equilibrium by co-evolutionary computation approach", IEEE Transaction on Power System, 24(3), pp. 1155-1164 (2009).

24. Hobbs, B., Metzler, C. and Pang, J. "Strategic gaming analysis for electric power systems: an MPEC approach", IEEE Transaction on Power System, 15(2), pp. 638-645 (2000).

25. 2006 Integrated Energy Plan: Parts 1-7. [Online]. Available: http://www.bchydro.com/info/reports.

26. Wen, F.S. and David, A.K. "Optimally coordinated bidding strategies in energy and ancillary service markets", Proc. Inst. Elect. Eng., Gener., Transm., Distrib., 149(3), pp. 331-338 (2002).

27. Bautista, G., Anjos, M.F. and Vannelli, A. "Numerical study of affine supply function equilibrium in $\mathrm{AC}$ network-constrained markets", IEEE Transaction on Power System, 22(3), pp. 1174-1184 (2007).

28. Rahimiyan, M. and Rajabi-Mashhadi, H. "An adaptive Q-learning algorithm developed for agent-based computational modeling of electricity market", IEEE Transaction on Power System, 40(5), pp. 547-556 (2010).

29. Lee, C. and Ou-Yang, C. "Development and evaluation 
of the interactive bidding strategies for a demander and its suppliers in supplier selection auction market", Int. J. Production Research, 46(17), pp. 4827-4848 (2008).

\section{Biographies}

Ahmad Azadi Hematabadi received his BS and MS degrees in Power Engineering from Mazandaran University, Iran, in 2003 and 2006, respectively. He is currently pursuing his $\mathrm{PhD}$ degree in the same field of study at Semnan University, Iran. His research interests are power system operation and restructuring.

Asghar Akbari Foroud received his BSc degree in Power Engineering from Tehran University and MSc and $\mathrm{PhD}$ degrees from Tarbiat-Modares University, Tehran, Iran, in 1997 and 2006, respectively. He is now working at Semnan University. His research interests include power system planning, dynamic \& operation, and restructuring. 